\title{
SPATIAL SOLITONS IN LIQUID CRYSTALS
}

\author{
M.A. KARPIERZ \\ Faculty of Physics, Warsaw University of Technology \\ Koszykowa 75, 00-662 Warszawa, Poland
}

\begin{abstract}
Reorientational phenomenon in liquid crystals is unique for nonlinear optics applications. Recently there have been shown that the reorientational nonlinearity in nematic liquid crystals can govern spatial solitons in both waveguide and bulk geometry. Such solitons require a few milliwats of light power and they can be controlled by the state of light polarisation or an external electric field. In this paper, the theoretical analysis and experimental results on optical solitons in nematic liquid crystals due to the reorientational nonlinearity are reviewed. The discussion of their properties is preceded by a brief introduction to nonlinear optics of liquid crystals.
\end{abstract}

\section{Introduction}

Liquid crystals are fascinating materials with many unique physical, optical, and electro-optical properties. New compounds and mixtures of liquid crystals are chemically stable, with low absorption, with very large optical anisotropy, with liquid crystalline phase in wide range of temperatures, are easily oriented at boundaries, and easily reoriented by electric or magnetic fields. Therefore, they are important optical materials for numerous applications in modern optoelectronics [1].

Liquid crystals are also excellent medium for nonlinear optics [1-3]. The main contribution to nonlinear optical phenomena in liquid crystals arises from thermal and reorientation processes. While the thermal effect is similar to that observed in other materials, the reorientation effect is characteristic only in liquid crystalline phases. The nonlinearity due to the reorientation effect in nematic phase leads to numerous effects not observed in another types of nonlinearity. The reorientation nonlinearity induces extremely large nonlinear changes of refractive index that can be obtained for relatively low light power. This nonlinearity depends on boundary conditions and geometry of the system and it can be easily modified by external electric or magnetic fields. The nonlinearity depends on light polarisation but within a wide range is independent on light wavelengths. The main drawback is slow response time, but by special optimisation of the system, this response could be fairly fast.

The nonlinear optics of liquid crystals has been a subject of intensive studies for more than twenty years. Most of the theoretical and experimental works were done for thin

A.D. Boardman and A.P. Sukhorukov (eds.), Soliton-driven Photonics, 41-57.

(C) 2001 Kluwer Academic Publishers 
samples and only a few papers reported propagation of light beam at lengths longer than the Rayleigh distance. There were experiments showing the self-focusing due to the reorientation process inside liquid crystals in capillaries [4-7], in planar cells [8], and in planar waveguides [9].

In this article, the main idea leading to create optical solitons due to the reorientation nonlinearity in liquid crystals is presented. Recently obtained experimental results and theoretical analysis are reviewed. First, in Section 2 the introduction to nonlinear optical effects in liquid crystals are briefly presented and in Section 3 the main properties of reorientation nonlinearity in nematic liquid crystals are shown. Next, the theoretical and experimental results in excitation of spatial solitons in waveguides (Section 4) and in bulk geometry (Section 5) are described. The concluding remarks and references close the paper.

\section{Optical nonlinearity in liquid crystals}

Liquid crystals (LCs) are composed of anisotropic molecules usually of a rod-like shape. In liquid crystalline phase the long-range interaction between molecules induces orientation ordering but allows flow of molecules. Therefore, they are fluid-like anisotropic materials with order characteristic for crystals and flow characteristic for liquids. Liquid crystalline phase is observed in some range of temperature of pure compounds and mixtures (for thermotropic LCs), in solutions (for lyotropic LCs) or for polymers. The main technical applications are connected with thermotropic LCs and their nonlinear properties are the aim of this paper.

There are several types of order observed in thermotropic LCs, usually classified as smectics, nematics, and their chiral forms (chiral nematics are called cholesterics). The simplest order is observed in nematic LCs where a position of molecules is arbitrary but the average orientation is determined. At a given temperature the nematic liquid crystal molecules fluctuate around the mean direction defined by the unity vector $\mathbf{n}$ called the director (see Fig. 1A). The orientation order is described by the parameter

$$
S=\frac{1}{2}\left\langle 3 \cos ^{2} \theta-1\right\rangle
$$

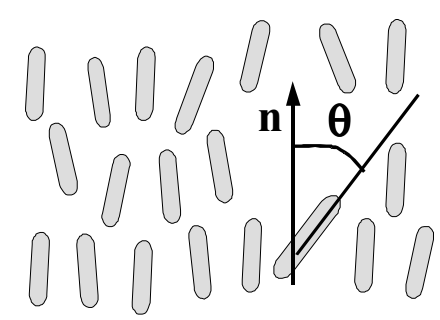

(A)

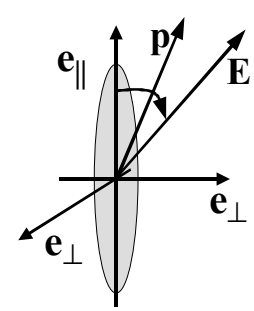

(B)

Figure 1. (A) Orientation of liquid crystalline molecules in nematic phase. (B) The dipole moment $\mathbf{p}$ induced by an electric field $\mathbf{E}$. 
where $\theta$ is an angle between molecule orientation and the director. The right side of the above definition is averaging over the time and space. For crystals the order parameter $S=1$, for isotropic liquids $S=0$ while for nematics usually $0.4<S<0.7$ and for smectics $S \approx 0.9$.

Liquid crystalline molecules are anisotropic and this is responsible for anisotropic properties of liquid crystals like the anisotropy of electrical pemittivity, magnetical permeability, coductivity, viscosity, optical birefringence etc. Particularly, electrical polarizability for field parallel to symmetry axis of a LC molecule is different than for fields perpendicular to the symmetry axis. Therefore, an external electric field $\mathbf{E}$ induces an electric dipole with the moment $\mathbf{p}$ that is not parallel to $\mathbf{E}$ (see Fig.1B). Consequently, a torque $\mathbf{p x E}$ tends to rotate molecule into alignment parallel with the applied electric field. Note, that reorientation does not depend on sign of the electric field and exists for time varying fields too. The interaction energy density connected with reorientation in electric field is given by:

$$
f_{e l}=-\frac{\varepsilon_{0} \Delta \varepsilon}{2}\left\langle(\mathbf{n} \cdot \mathbf{E})^{2}\right\rangle,
$$

where $\Delta \varepsilon=\varepsilon_{\|}-\varepsilon_{\perp}$ is an electrical anisotropy, $\varepsilon_{\|}$and $\varepsilon_{\perp}$ are respectively the extraordinary and the ordinary components of the electric permittivity. This energy is minimised when the molecules are parallel to the electrical fields (for positive anisotropy $\Delta \varepsilon>0$ ). Similar behaviour is observed for magnetic fields but magnetic anisotropy is usually much lower than electrical one. For optical frequencies, the interaction with the magnetic field can be neglected and the interaction between light and liquid crystal is described as an interaction with the electric field.
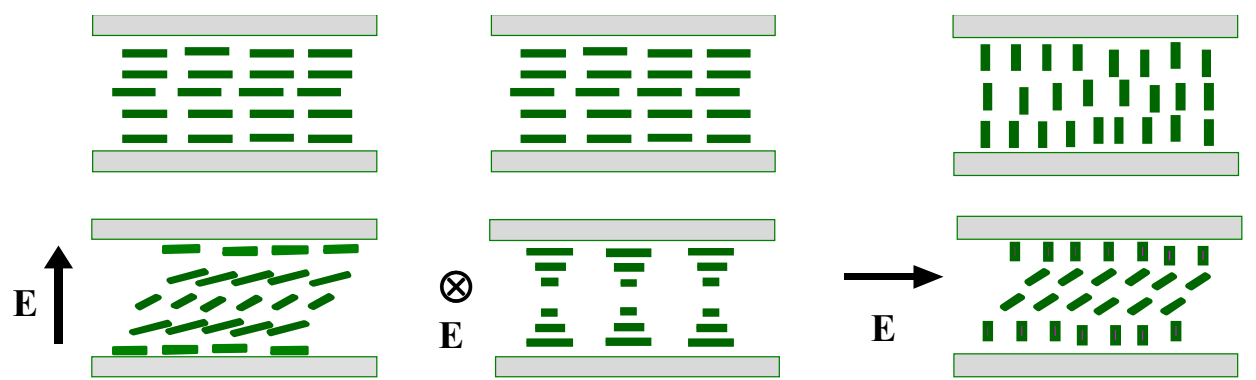

Figure 2. Basic deformations induced by an electric field E: splay (left), twist (centre) and bend (right) respectively

Anchoring conditions at the boundaries induce orientation in the liquid crystalline cell. This orientation is restrained by elastic forces. Any change of the orientation (e.g. induced by an electric field) is connected with superposition of three basic deformations presented in Fig.2. Distortion of the molecular alignment corresponds to the free energy density: 


$$
f_{F}=\frac{1}{2} K_{11}(\operatorname{div} \mathbf{n})^{2}+\frac{1}{2} K_{22}(\mathbf{n} \cdot \operatorname{rot} \mathbf{n})^{2}+\frac{1}{2} K_{33}(\mathbf{n} \times \operatorname{rot} \mathbf{n})^{2}
$$

where $K_{\mathrm{ii}}$ are elastic constant for three types of deformation: splay (i=1), twist (i=2), and bend ( $\mathrm{i}=3$ ). Usually $K_{33}>K_{11}>K_{22}$ and they are of order of magnitude $10^{-11} \mathrm{~N}$. In theoretical analysis equations are simplified by assumption that all elastic constants are equal (i.e. $K_{11} \approx K_{22} \approx K_{33}$ ).

The orientation in liquid crystalline cell is a result of interaction with external fields and boundaries. Light wave can also introduce changes of LC initial orientation. Because the birefringence of LC is connected with the orientation of molecules, the changes of orientation cause the rotation of the optical birefringence axis. It means that the light modifies the electric permittivity tensor. This is a mechanism of the reorientation nonlinearity. The liquid crystal anisotropy is relatively large (even $\Delta \varepsilon \sim 0.6$ ) and therefore the reorientation nonlinearity creates giant refractive index changes.

To calculate the reorientation phenomena the minimisation of the free energy is used. The total free energy density includes the deformation energy $f_{\mathrm{F}}$ and the energy of interaction with external fields as well as with boundaries. The minimisation procedure leads to the Euler-Lagrange equations. It is convenient to introduce the orientation angle $\theta$ between the director $\mathbf{n}$ and one of the co-ordinates. The angle $\theta$ is sufficient to describe the orientation in the two-dimensional problem i.e. where the liquid crystal molecules at the boundaries and the electric field are in the same plane. Then the equation in order to one unknown function $\theta$ is taken into calculations.

In liquid crystals are also observed other mechanisms of optical nonlinearity like electronic, thermal, photorefractive or electrostrictive. The largest contribution to nonlinear effects is caused by thermal and reorientational mechanisms. Recognition, which mechanism is the source of observed effects, is important problem in many experiments. It should be pointed that the temperature changes LC density but also the order parameter $S$ (it causes that typically the ordinary refractive index increases with increasing temperature while the extraordinary refractive index decreases with increasing temperature). Generally, the thermal nonlinearity in LCs has similar properties like in other anisotropic materials. The reorientational nonlinearity however is characteristic only for liquid crystals and it does not exists in other materials. In this paper only the mechanism of reorientational nonlinearity will be analysed

Nonlinear properties connected with reorientational phenomena are dependent on initial orientation and therefore they depend on LC cell configuration. For the self-focusing phenomena (and spatial solitons), two simplest cells were used: LC in capillary or between two plates (see Fig.3). If the LC molecules at boundaries are oriented in perpendicular way then in a thick capillary, they create the axial texture (Fig. $3 \mathrm{~A}$ ) and in a planar cell, they create the homeotropic texture (Fig.3C). For parallel orientation of LC molecules at boundaries, the planar texture is achieved (Fig.3B and Fig.3D).

If transversal dimensions of the cell are much larger than the wavelength and the size of the input light beam then LC can be treated as a bulk medium. Such configuration is proper for observation of $(2+1)$ dimensional solitons. When the distance between bounded walls is comparable with the wavelength and the beam dimension then a liquid crystalline cell should be treated as a planar waveguide or an optical fibre [10-11]. In planar waveguides $(1+1)$ dimensional solitons could be formed. 


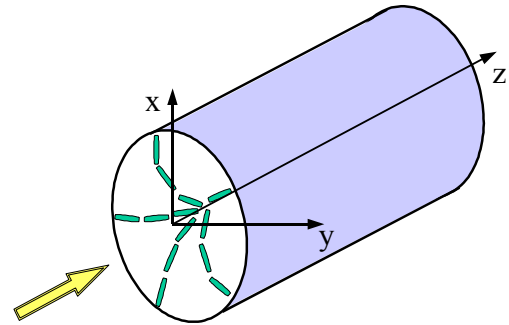

(A)

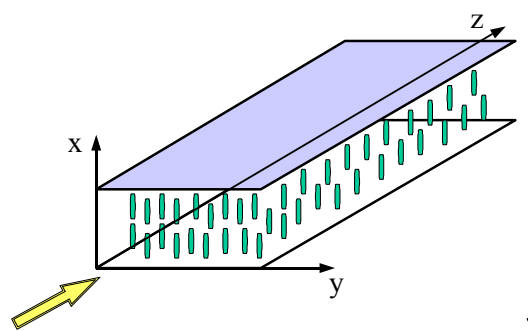

(C)

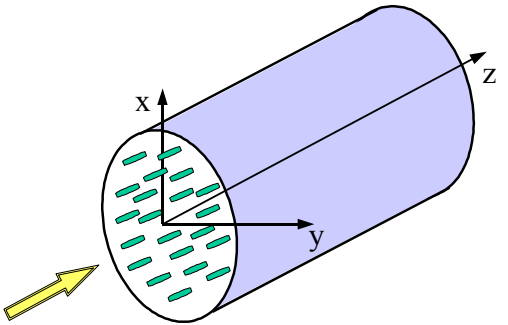

(B)

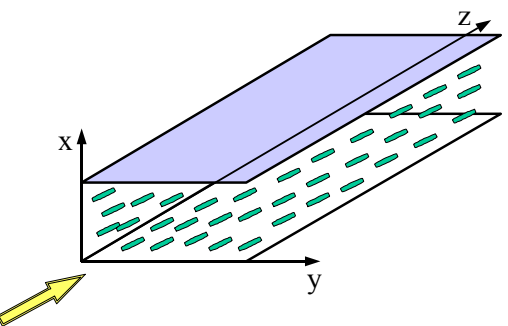

(D)

Figure 3. Configurations of LC cells: (A) capillary with axial texture, (B) capillary with planar texture, (C) planar with homeotropic texture, (D) planar with planar texture

\section{Liquid crystalline planar waveguides}

An influence of the initial LC texture and light beam parameters on nonlinearity will be illustrated for modes of planar waveguides [12-14]. In planar waveguides, the liquid crystalline layer can play a role of a waveguide core or a waveguide cover. Initially the liquid crystal can be oriented in different ways and these different orientations are forced at boundaries. Optical nonlinear effect strongly depends on the used initial orientation and on the light polarisation. For example, in the homeotropic alignment from Fig. $3 \mathrm{C}$ the $E_{\mathrm{x}}$ component of the electric field is not able to reorient the liquid crystal molecules. Therefore, such waveguide configuration is linear for the light polarised in $x$ direction.

The configuration as in Fig.4 is assumed with both the director $\mathbf{n}$ and an electric field $\mathbf{E}$ parallel to $x z$ plane. Then the orientation of LC molecules is defined by the orientation angle $\theta$ and the director $\mathbf{n}=(\cos \theta, 0, \sin \theta)$. For the one elastic constant approximation (i.e. for $\mathrm{K}=\mathrm{K}_{11} \approx \mathrm{K}_{22} \approx \mathrm{K}_{33}$ ) the Euler-Lagrange equations have the form:

$$
\frac{\partial^{2} \theta}{\partial x^{2}}+\frac{\partial^{2} \theta}{\partial y^{2}}+\frac{\partial^{2} \theta}{\partial z^{2}}+\frac{\varepsilon_{0} \Delta \varepsilon}{4 K}\left(\left(E_{x} E_{z}^{*}+E_{x}^{*} E_{z}\right) \cos 2 \theta+\left(\left|E_{z}\right|^{2}-\left|E_{x}\right|^{2}\right) \sin 2 \theta\right)=0 .
$$




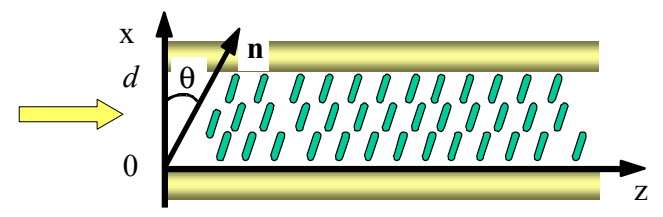

Figure 4. Configuration of the analysed LC planar waveguide

The equation (3) with boundary conditions $\theta(x=0)$ and $\theta(x=d)$ describes the reorientation of the liquid crystal due to the electric field of the electromagnetic wave. The reorientation changes the birefringence axis and the electric permittivity tensor has the form:

$$
\varepsilon=\left(\begin{array}{ccc}
\varepsilon_{\perp}+\Delta \varepsilon \cos ^{2} \theta & 0 & \Delta \varepsilon \sin \theta \cos \theta \\
0 & \varepsilon_{\perp} & 0 \\
\Delta \varepsilon \sin \theta \cos \theta & 0 & \varepsilon_{\perp}+\Delta \varepsilon \sin ^{2} \theta
\end{array}\right)
$$

The Maxwell's equations in anisotropic dielectric medium complete the set of equations theoretically describing the system. Note that the configuration taken into consideration is proper for TM electromagnetic waves with non-vanished $E_{\mathrm{x}}, H_{\mathrm{y}}$ and $E_{\mathrm{z}}$ field components. For planar waveguide modes, the field independent on transversal $y$ direction is assumed:

$$
\begin{aligned}
& E=E(x) \exp \left[\mathrm{i} \omega t-\mathrm{i} k_{0} N z\right], \\
& H=H(x) \exp \left[\mathrm{i} \omega t-\mathrm{i} k_{0} N z\right],
\end{aligned}
$$

where $N$ is the effective refractive index, and the reorientation introduced by such field depends only on $x$ : $\theta(x)$. Then the mathematical description of the problem consists of the equation (3) without second and third differential components and the Maxwell's equations for the field (5)-(6) in media with electrical permittivity (4). Because of the complexity of the equation system, the solution can be obtained only by using numerical methods.

The mechanism of the nonlinear guided wave formation is following the scheme: a guided field reorients liquid crystal that causes reorientation of the local optical axis and this modifies the guided field shape. In figure 5A the guided TM mode profile $H_{\mathrm{y}}(x)$ is presented for the initial homeotropic orientation $\theta(x)=\pi / 2$ (curve '1') and for the light power strong enough to reorient liquid crystalline layer (curve ' 2 ' and corresponding to this field the orientation angle $\theta(x))$. All theoretical curves presented in this article were obtained for parameters corresponding to 6CHBT (4-trans-4'-n-hexyl-cyclohexylisothiocyanatobenzene) nematics: $\varepsilon_{\|}=(1.69)^{2}, \varepsilon_{\perp}=(1.52)^{2}$ for wavelength $\lambda=842 \mathrm{~nm}$.

Due to the large anisotropy of LCs, the guided mode is changing its profile significantly, when LC reorients (compare curves ' 1 ' and '2' in Fig.5A). Together with changes of the field profile, the effective refractive index is changing with increasing of 
the light power. The effective index versus the power of the guided mode is plotted in the Fig.5B. The unit of dimensionless power density in $y$-direction used in this paper corresponds roughly to $2 \mathrm{~mW} / \mu \mathrm{m}$ for typical LC waveguide structures.
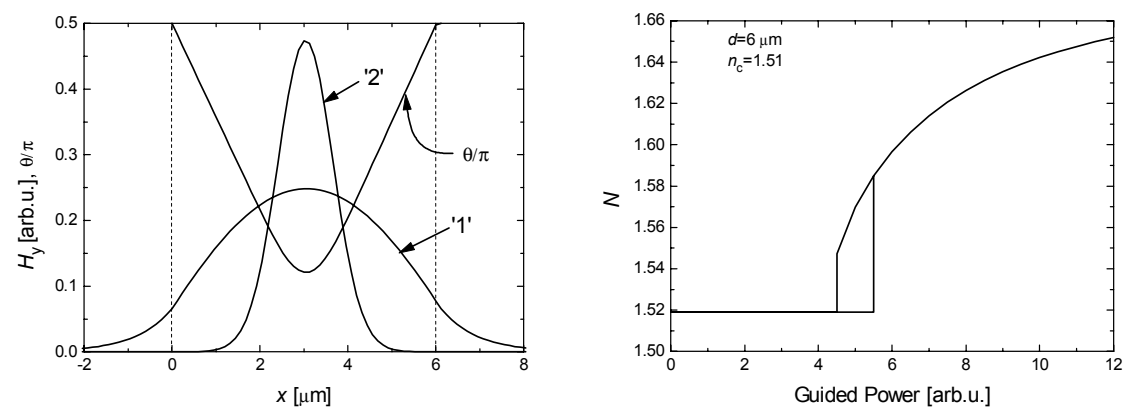

(A)

(B)

Figure 5. (A) Distribution of $H_{\mathrm{y}}(\mathrm{x})$ in guided mode without reorientation (curve '1') and with reoriented LC film (curve ' 2 ') and corresponding to this case the orientation angle $\theta(\mathrm{x})$; (B) effective refractive index vs. light power. Both plots were obtained for film thickness $d=6 \mu \mathrm{m}$ and refractive index of surrounding plates $n_{\mathrm{c}}=1.51$

The characteristics presented in Fig.5B shows that the nonlinear change of the effective index is bistable and begins above the threshold value of the light power. Optical bistability is caused by large nonlinearity and the threshold character of the reorientation. The reorientational effect depends not only on the local value of the electric field but on the electric field profile in a cross-section. On the other hand, changes of the orientation induce changes of the field profile of the guided waves. These field profile changes due to the nonlinear reorientation are very large. Therefore, the power value of field too week to introduce reorientation can be the same as the power value of field existing in reoriented structure. Non-local nature of the nonlinear reorientational effect is a source of a feedback, which is necessary in optical bistability. When the electric field tends to reorient liquid crystalline molecules positioned at an angle $\pi / 2$ the reorientation starts above the threshold value of the electric field. This phenomenon is called the threshold Freedericksz transition effect. The origin of this effect is a fact that the torque rotating LC molecules does not exist for $\mathbf{E} \perp \mathbf{e}_{\|}$(see Fig.1B). Then the reorientation can start due to fluctuations of the molecular orientation but it requires electrical field strong enough.

The existence of the Freedericksz effect can be also explained as follows. For initial planar orientation in LC layer $(\theta=\pi / 2)$ the beginning of reorientation can be analysed in term of a small angle $\delta=\theta-\pi / 2<<1$. Then neglecting the longitudinal part of the electric field $\left(E_{\mathrm{z}}<<E_{\mathrm{x}}\right)$ the equation (3) is simplified to the form:

$$
\frac{d^{2} \delta}{d x^{2}}+\frac{\varepsilon_{0} \Delta \varepsilon}{2 K}\left|E_{x}\right|^{2} \delta=0
$$


The above equation (7) is formally equivalent to the Schrödinger equation for particle in a potential well (difference between particle energy and potential is defined by $\left|E_{\mathrm{x}}(x)\right|^{2}$ ) with infinite walls (because of the boundary conditions $\delta(x=0)=\delta(x=d)=0$ ). The lowest eigenstate of this problem requires that energy should be larger than potential. This means that for nonzero solution the value of $\left|E_{\mathrm{x}}(x)\right|^{2}$ has to be larger than some threshold value, corresponding to minimal value of the energy. For lower values of $\left|E_{\mathrm{x}}(x)\right|^{2}$ only the solution $\delta=0$ exists, that means no reorientation.

The sharp threshold disappears if the reorienting field is not perpendicular to the director. Consequently, the optical bistability of effective refractive index disappears. In Fig.6A the nonlinear characteristics are presented for the nematics tilted at the boundaries: $\theta(x=0)=\theta(x=d)=\theta_{0}$. Note, that by changing the initial tilt $\theta_{0}$ it is possible to modify the nonlinearity (from bistable, with threshold to very smooth).

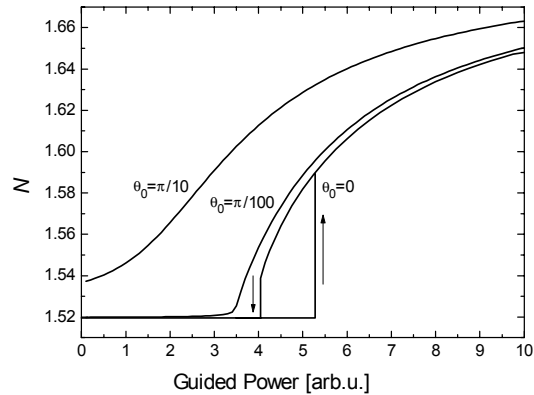

(A)

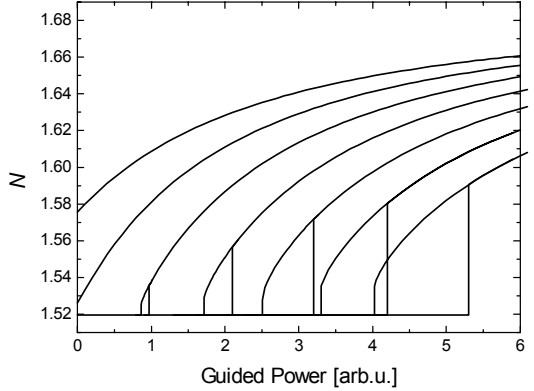

(B)

Figure 6. Effective refractive index vs. the power of the guided mode: (A) for different tilt angle at boundaries in tilted nematics configuration, (B) for different value of external electric field applied to the LC layer (upper characteristics were obtained for higher strength of the external field)

The nonlinear characteristics could be also changed by external slowly varying electrical fields applied to the LC film (see Fig.6B). The homogenous external field can support the optical field and shift the nonlinear characteristics towards lower power and smoothing their shape.

Reorientational nonlinearity is a source of very large refractive index changes induced by relatively low values of the light intensity. To observe significant nonlinear effects usually less than a few $\mathrm{kW} / \mathrm{cm}^{2}$ is sufficient. From theoretical curves plotted in figures 5 and 6 approximately $10 \mathrm{~mW} / \mu \mathrm{m}$ of light power in a planar mode induces effective index changes of a value $\Delta n \sim 0.1$. This extremely large nonlinearity (by some authors called giant) can be enhanced even by two orders of magnitudes in a presence of dyes in a LC mixture [15]. This effect discovered by Janossy is strongly dependent on the light wavelength (see Fig. 7) and can lead to diminishing nonlinearity as well as to changing the sign of reorientation. It should be pointed out that the Janossy effect has not thermal but absorptive origin. 


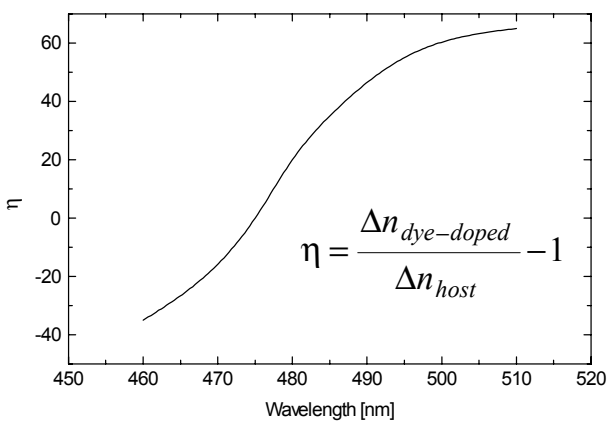

Figure 7. Typical dependence of the enhancement parameter $\eta$ on the wavelength in the Janossy effect

The main drawback of the reorientational nonlinearity in nematics is a slow response time, which could be of order of seconds. The time of the reorientational process depends among others on LC viscosity, values of the elastic constants, thickness of a layer, strength of reorienting fields. However, in constructions of the electro-optical liquid crystalline displays the response time of reorientation were improved to a few microseconds (for ferroelectric displays). Therefore it can be expected that optimisation of all-optical nonlinear devices will also decrease the response time of the reorientational nonlinearity.

Concluding properties of the reorientational nonlinearity in nematic LCs, this kind of nonlinearity is unique in many aspects. Particularly nonlinear characteristics can be fitted to required form by special preparing of the initial orientation in the sample. They can be also externally controlled by electric or magnetic fields. This nonlinearity is dependent on light polarisation and could have threshold as well as bistable form. Because of the nature of orientation, changes induced by one beam are recognised by any other in wide range of wavelengths. Finally, reorientational optical nonlinearity is extremely large and can be additionally enhanced by special dye-doping.

\section{Solitons in liquid crystalline waveguides}

Planar waveguide structures confine fields in one transversal direction and therefore are proper media to observe $(1+1)$ dimensional spatial solitons. In LC waveguides such solitons were analysed and measured in configuration with homeotropicaly aligned nematics (Fig.3A) [9,13].

Guided fields in anisotropic waveguides are generally hybrid but they can be superposed of TE-like and TM-like guided modes, i.e. with dominating $E_{\mathrm{x}}$ component (for TM-like modes) and with dominating $E_{\mathrm{y}}$ component (for TE-like modes) of electric field. Therefore, in analysed homeotropically aligned LC waveguide the light beam consists of two perpendicularly polarised TE-like and TM-like fields (see Fig.8A). 


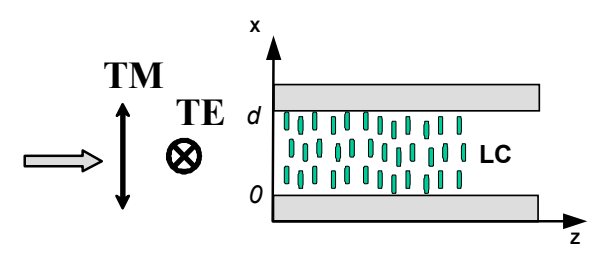

(A)

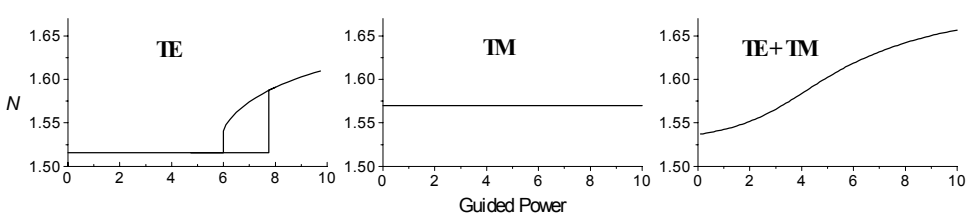

(B)

Figure 8. Analysed configuration of LC waveguide and corresponding effective index changes for different light polarisation

If the light is TE-like polarised, i.e. an electric field is perpendicular to the initial orientation of LC molecules, reorientational nonlinearity has the threshold and can be bistable (like in Fig.5). On the other hand for the TM-like polarisation, i.e. with an electric field parallel to the LC molecule orientation, the reorientational nonlinearity does not appear. Combination of both TE and TM fields gives the nonlinear response without threshold. This nonlinearity is dependent on the state of polarisation i.e. dependent on phase differences and amplitudes ratio between TE and TM fields. Concluding, in analysed waveguide different nonlinear refractive index changes for different light polarisations can be obtained, what is schematically plotted in Fig.8B.

Stable solitons in $(1+1)$ dimensions exist if the nonlinear change of refractive index is weaker than a linear function of the light intensity. From that point of view the selffocusing of pure TE-like field in analysed structure is unstable. Therefore, the light beam with both TM and TE fields but with stronger TM-like component will be considered. Then the nonlinear effect will start without threshold and will be low enough to neglect changes of the field profile in guide modes. Consequently, the electric field components of light beam can be described as:

$$
\begin{aligned}
E_{x} & =A(y, z) \psi(x) \exp \left(i \omega t-i k_{0} N_{x} z\right), \\
E_{y} & =B(y, z) \varphi(x) \exp \left(i \omega t-i k_{0} N_{y} z\right),
\end{aligned}
$$

where $\psi(x) \exp \left(\mathrm{i} \omega t-\mathrm{i} k_{0} N_{\mathrm{x}} z\right)$ and $\varphi(x) \exp \left(\mathrm{i} \omega t-\mathrm{i} k_{0} N_{\mathrm{y}} z\right)$ are TM and TE modes of the planar waveguide with corresponding effective refractive indices $N_{\mathrm{x}}$, and $N_{\mathrm{y}}$. Complex amplitudes $A$ and $B$ are slowly varying in respect to $z$ and fulfill the relation $|A|>>|B|$. Starting from Maxwell's equations for fields in the form (8)-(9) with some simplifying assumptions the pair of equations for amplitudes $A$ and $B$ are obtained: 


$$
\begin{aligned}
& {\left[\kappa_{1}+\frac{1}{2 k_{0} N_{y}} \frac{\partial^{2}}{\partial y^{2}}-i \frac{\partial}{\partial z}\right] B=-\kappa_{12} A \exp \left(-i k_{0}\left(N_{x}-N_{y}\right) z\right),} \\
& {\left[-\kappa_{2}+\frac{1}{2 k_{0} N_{x}} \frac{\partial^{2}}{\partial y^{2}}-i \frac{\partial}{\partial z}\right] A=-\kappa_{12} B \exp \left(i k_{0}\left(N_{x}-N_{y}\right) z\right),}
\end{aligned}
$$

where $\kappa_{1} \sim \int \sin ^{2} \theta \varphi^{2} d x, \kappa_{2} \sim \int \sin ^{2} \theta \psi^{2} d x, \kappa_{12} \sim \int \sin \theta \cos \theta \varphi \psi d x$ are coefficients dependent on LC orientation. The coefficient $\kappa_{12}$ is responsible for a coupling between TE and TM waves. The coefficients $\kappa_{1}$ and $\kappa_{2}$ effectively modify the propagation constants of both fields. For pure homeotropic alignment $(\theta(x)=0)$ all coefficients are equal to zero and both fields propagate independently with different phase velocities. Diffraction of such beams is presented in fig.9. When the light intensity increases the nonlinear reorientation appears and this induces interaction between both field component and modification of light propagation.

Orientation angle $\theta$ changed by the light beam is calculated from a formula similar to the equation (3):

$$
\frac{d^{2} \theta}{d x^{2}}+\frac{\varepsilon_{0} \Delta \varepsilon}{4 K}\left[2|A B| \varphi \psi \cos \Delta \alpha \cos 2 \theta+\left(|B \varphi|^{2}-|A \psi|^{2}\right) \sin 2 \theta\right]=0,
$$

where $\Delta \alpha$ is the phase difference between $E_{\mathrm{x}}$ and $E_{\mathrm{y}}$ components. Equation (12) allows to calculate the nonlinear coefficients $\kappa_{1}, \kappa_{2}$ and $\kappa_{12}$ which can be approximated with a very high accuracy by functions:

$$
\begin{gathered}
\kappa_{12} \sim \frac{|A B| \cos \Delta \alpha}{\left(1+\left|A / A_{S}\right|^{2}\right),} \\
\kappa_{1,2} \sim \frac{|A B|^{2} \cos ^{2} \Delta \alpha}{\left(1+\left|A / A_{S}\right|^{2}\right)^{2}},
\end{gathered}
$$

where $A_{\mathrm{S}}$ is a saturation amplitude.

Note, that for $\Delta \alpha=0$ the reorientation is the largest while for $\Delta \alpha=\pi / 2$ it disappears (because $|A|>>|B|)$. The phase difference $\Delta \alpha$ in linear case is equal to $k_{0}\left(N_{\mathrm{x}}-N_{\mathrm{y}}\right) z$ and this causes changes of the light polarisation with a period equal to the birefringence length $L_{\mathrm{B}}=\lambda /\left(N_{\mathrm{x}}-N_{\mathrm{y}}\right)$ which in LC could be as low as a few wavelengths. Consequently, the nonlinear changes of LC orientation should be periodic with a spatial period in first approximation equal to $L_{\mathrm{B}}$.

Equations (10)-(11) with coefficients in the form (13)-(14) are a basis of the theoretical analysis of beam propagation in waveguides with homeotropically aligned nematics. In figure 10 the self-trapped beam formation is presented in a waveguide of thickness $10 \mu \mathrm{m}$ filled with nematic $6 \mathrm{CHBT}$. At the input, the light beam with a gaussian-like shape of the width $\sim 10 \mu \mathrm{m}$ is launched with 10 times larger intensity of the TM-like 
component than the TE-like component. For low intensity, the linear diffraction is obtained like in Fig. 9.

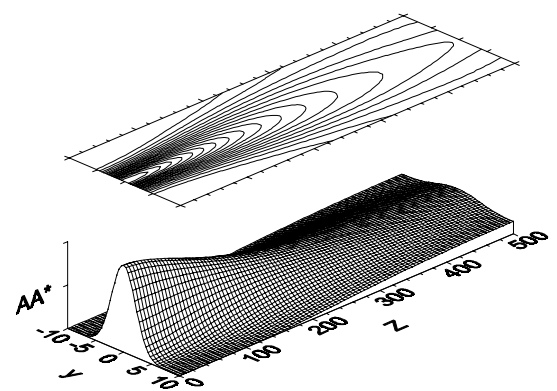

(A)

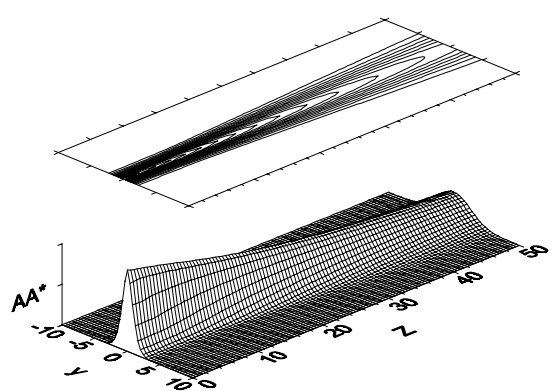

(B)

Figure 9. Diffraction of light beams with two initial widths. Dimensions are in micrometers and the light intensity is in arbitrary units

For power approximately $30 \mathrm{~mW}$, the light forms the spatial solitary wave that is stable at longer distances. The TM-polarised component of formed soliton has the size approximately twice larger than the TE-polarised component. Generally, the size of both polarisation components is different and it depends on ratio between their intensities. Note that beams with dimension corresponding to TM and TE-polarised component of the self-trapped beam presented in Fig.10 should diffract in linear case like beams presented respectively in Fig.9A and Fig.9B.

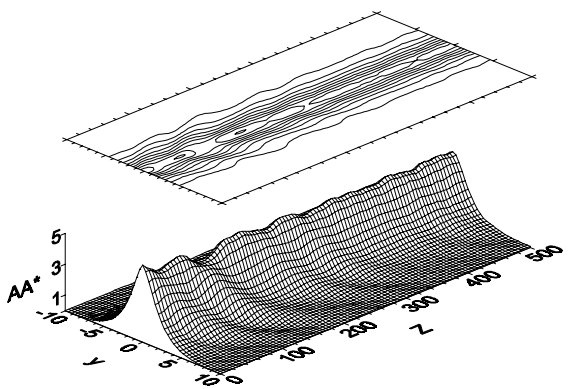

(A)

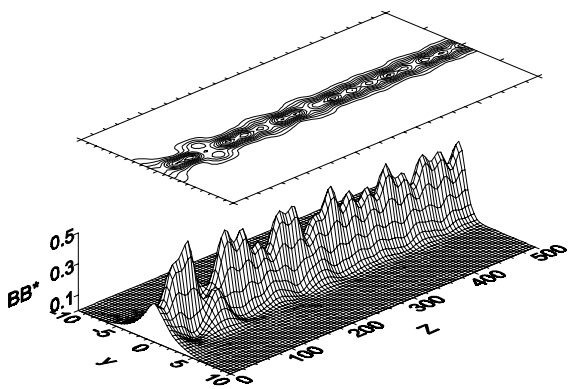

(B)

Figure 10. Self-focusing of the light beam in LC waveguide: (A) TE polarisation component, (B) TM polarisation component. Initially the field distribution in both polarisations has the same width and the intensity of the light in TE-like field is 10 times larger than in TM-like field

Obtained spatial soliton is formed by the periodically changed reorientation what is presented in Fig.11. This periodical modulation of reorientation is induced by interfering components of the guided beam and it traps the light beam not allowing it to diffract. 


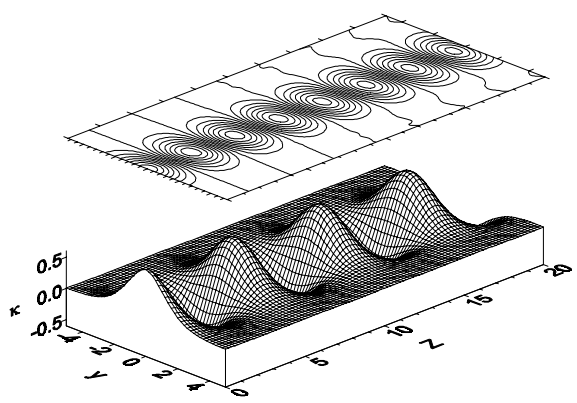

Figure 11. Reorientation coefficient $\kappa_{12}$ corresponding to the soliton formed in Fig. 10 at the end of propagation distance

The forms of the nonlinear coefficients (13)-(14) suggests that properties of obtained solitons are similar to solitons in saturable nonlinearity (like photorefractive screening solitons) [16]. In fact, the set of equations is different than usually analysed but generally, they are vectorial solitons in anisotropic medium with the anisotropic saturation-like nonlinearity.

Self-focusing phenomena in LC waveguides has been observed in experimental set-up which is shown schematically in fig. 12. 6CHBT nematic LC created $10 \mu \mathrm{m}$ homeotropically aligned film between two glass plates. Light at wavelength $\lambda=842 \mathrm{~nm}$ from the laser diode pigtailed with the single-mode fibre was launched symmetrically to the LC film. Propagation of the light beam in the LC waveguide was observed through the scattered light detected by a CCD camera. Obtained photographs are presented in Fig. 13. For low values of light power, $(P<<20 \mathrm{~mW})$ diffraction of light was detected. For the light power $\mathrm{P} \approx 20 \mathrm{~mW}$ but for the TE-like polarisation the self-focusing is found to be unstable. Finally, for the light power $\mathrm{P} \approx 30 \mathrm{~mW}$ but for dominating the TM-like polarisation the stable self-trapped beam is observed.

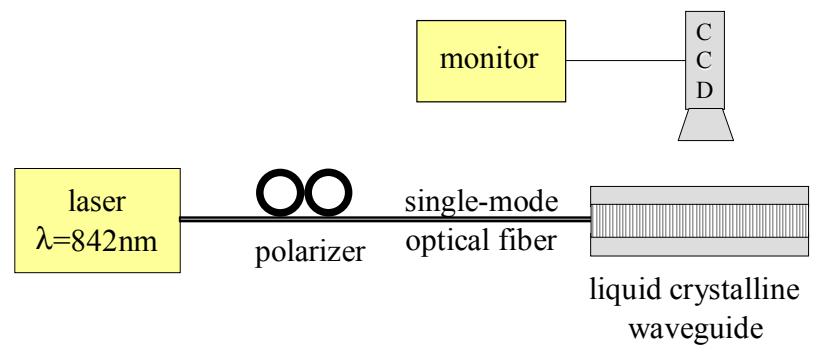

Figure 12. Experimental set-up for observation of self-focusing in LC cell 

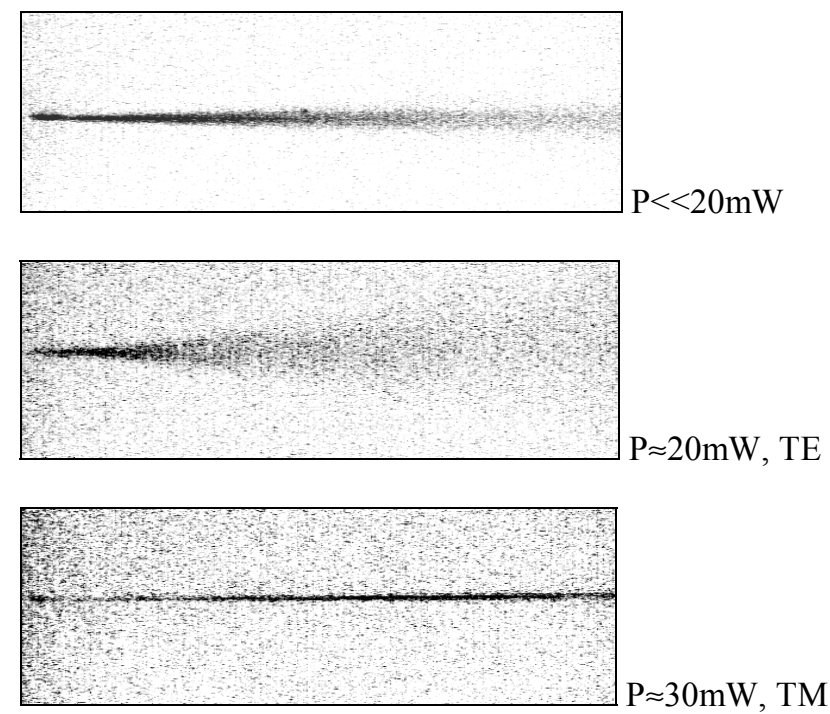

Figure 13. Experimental results obtained in a planar waveguide [9]: light scattered from LC waveguide for linear case (top), with dominant TE-like field (middle) and for dominant TM-like field (bottom). The length of the photographs is approximately $0.6 \mathrm{~mm}$ that correspond to more than 3 diffraction lengths

In the case when the spatial soliton was formed, the birefringence of the sample was also measured. It was found that $\mathrm{LC}$ molecules in the beam area rotate with spatial period corresponding to the birefringence length (see Fig. 14). It is in a perfect agreement with theoretical predictions and it proves that the reorientation is the source of the beam self-trapping.

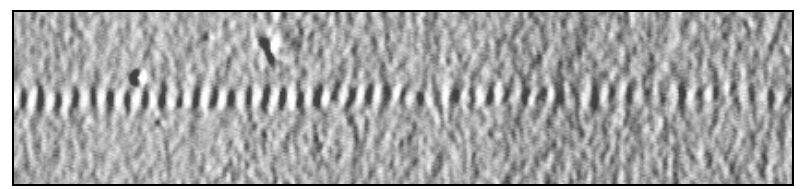

Figure 14. Birefringence of the $\mathrm{LC}$ waveguide with the presence of optical soliton in the central part. Pattern achieved in white light passed two crossed polarizers. Lighter areas correspond to rotation of LC molecules in one direction while darker areas correspond to rotation in opposite direction

\section{Solitons in bulk nematics}

When the transversal size of the liquid crystalline cell is larger than the beam size the LC can be treated as a bulk medium. The size of the cell cannot be very large because of difficulties to obtain the homogenous orientation in thick layers (usually cell dimension is limited to hundreds of micrometers). Although the light beam is concentrated in a small area the reorientation has a non-local character and can appear far from the light 
beam. Therefore, the shape of the cell and the orientation on the boundaries are important.

The beam propagation in a bulk medium should be described by the three-dimensional wave equation, which in scalar paraxial form is equal to:

$$
2 i k_{0} \frac{\partial E}{\partial z}+\frac{\partial^{2} E}{\partial x^{2}}+\frac{\partial^{2} E}{\partial y^{2}}+k_{0}^{2} \Delta \varepsilon \sin ^{2} \theta=0
$$

The reorientation of LC is described by the Euler-Lagrange equation, which for the oneelastic constant approximation and for the scalar field has the form:

$$
\frac{\partial^{2} \theta}{\partial x^{2}}+\frac{\partial^{2} \theta}{\partial y^{2}}+\frac{\varepsilon_{0} \Delta \varepsilon}{2 K}|E|^{2} \sin 2 \theta=0
$$

The above theoretical model neglects any polarisation effects and is valid for a small reorientation. However, obtained results are in good agreement with experiments $[8,17,18]$.

$(2+1) \mathrm{D}$ self-focusing of the light beam in bulk nematics were reported both in the capillary with planar texture (fig.3B) and in the planar cell with planar texture (fig.3D). In experiments done by Braun et al. [11,12], the nematic liquid crystal E206 (commercial compound) was inserted in a glass capillary of inner diameter $1.5 \mathrm{~mm}$. Light beam from an argon laser was focused at the input to a spot of size around $50 \mu \mathrm{m}$. The beam was observed by looking at the scattered light with the microscope (in similar way as in the set-up from Fig.13). The light polarisation was perpendicular to the director of LC and thus the reorientation was achieved above Freedericksz threshold. As the light power was increased first the focal spots were observed (for intensities $I \sim 0.6$ $\mathrm{kW} / \mathrm{cm}^{2}$ ). Then, the light beam off-axis meandered (undulation for intensity I 1.9 $\mathrm{kW} / \mathrm{cm}^{2}$ ) and broke-up (for $\mathrm{I} \sim 2 \mathrm{~kW} / \mathrm{cm}^{2}$ ) in a form of two distinct filaments undulating in phase opposition. Because of threshold nonlinearity there were not observed selftrapped spatial solitons. Detailed theoretical studies of the experiment were presented by McLaughlin et al. $[17,18]$.

Similar results were obtained by Warenghem et al. [6,7]. In that experiment, the light from argon laser was launched to the capillary cell through the optical fibre. This allowed obtain at the input beam size about $10 \mu \mathrm{m}$ and $4 \mu \mathrm{m}$. In a capillary of diameter $250 \mu \mathrm{m}$ nematic 5CB (4-cyano-4'-n-pentylbiphenyl) was doped with small amount of an anthraquinone dye (AQ1) to enhance the reorientational effect due to the Janossy effect. The nonlinear self-focusing was observed for a few $\mathrm{mW}$ of light power. Additionally to events reported previously, the appearance of a "self waveguiding" structure was observed.

Very recently in the work by Peccianti et al. [8] the stable spatial solitons were observed in the planar cell. To eliminate threshold in nonlinear response an external voltage was applied to the cell. Light beam from an argon laser was collimated to the waist $<2.5 \mu \mathrm{m}$ and launched to the $75 \mu \mathrm{m}$ thick layer with E7 (commercial mixture) nematic LC. Without external electric field the light beam was strongly self-focused that 
resulted in instabilities and beam break-ups (see fig.15). External electric field induced initial reorientation that caused optical nonlinearity without threshold. As a consequence the light beam was stable self-trapped and optical soliton was formed. The reorientation origin of this effect was proved by using a weak collinear He-Ne probe that was guided by the soliton only if copolarized with it.

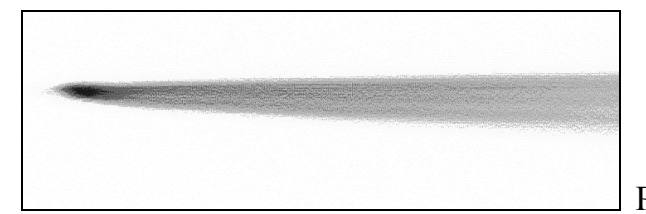

$\mathrm{P}=0.1 \mathrm{~mW}, \mathrm{U}=0 \mathrm{~V}$
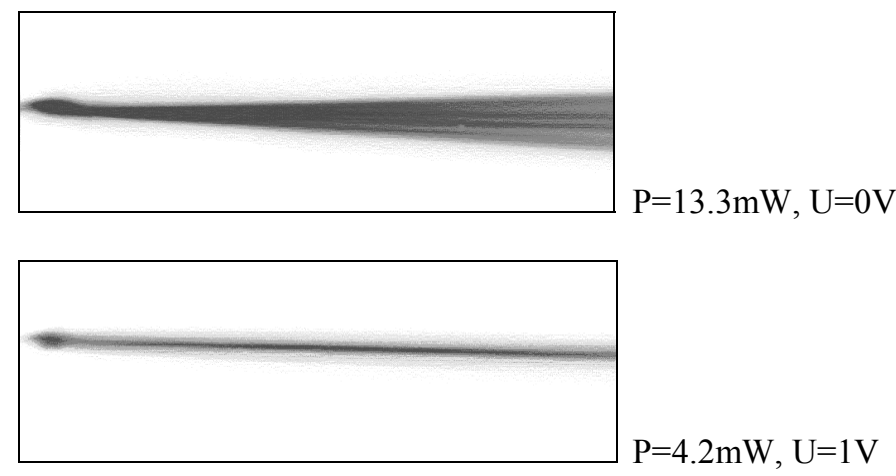

Figure 15. Experimental results obtained in planar cell [8]: light scattered from LC planar cell for low power (top), without external field (middle) and with external electric field (bottom). The length of the photographs is approximately $1 \mathrm{~mm}$ (with permission of Prof. G. Assanto)

\section{Conclusions}

Nematic liquid crystals seem to be very interesting medium for creation of optical spatial solitons. Experimental results showed that for light power of the order of only a few $\mathrm{mW}$ can be achieved self-trapped beam at distances of the order of a few $\mathrm{mm}$. The stability of such beams can be controlled by external fields or state of the light polarisation. The achieved solitons require very low light power but the relaxation time of their creation is very long. However, investigations of optical spatial solitons in liquid crystals are at an early stage. The used configurations are not optimised and probably like in LC displays, the response time could be shortened to a few $\mu$ s. Shorter response time is possible for others nonlinear mechanisms in LCs and e.g. for thermal nonlinearity it could be as low as a few ns.

The progress in the synthesis of new liquid crystals and their use in optoelectronic devices and particularly in nonlinear optics is extremely rapid. Therefore, significant progress in liquid crystalline optical solitons can be expected in the near future. Especially, new configuration and sample geometries as well as new types of liquid crystals like ferroelectric or polymeric could be used. 


\section{References}

1. I.C. Khoo, N.T. Wu, Optics and nonlinear optics of liquid crystals, World Scientific Publishing, Singapore, New Jersey, London, Hongkong 1993.

2. N.V. Tabiryan, A.V. Sukhov, B.Ya. Zeldovich, "The orientational optical nonlinearity of liquid crystals," Mol. Cryst. Liq. Cryst. 136 (1986) 1-139.

3. I.C. Khoo, "Nonlinear optics of liquid crystals," Progress in Optics XXVI, ed. E. Wolf, Elsevier Science Publishers 1988, 105-161.

4. E. Braun, L.P. Faucheux, A. Libchaber, D.W. McLaughlin, D.J. Muraki, M.J. Shelley, "Filamentation and undulation of self-focused laser beams in Liquid crystals", Europhys. Lett. 23 (1993) 239-244.

5. E. Braun, L.P. Faucheux, A. Libchaber, "Strong self-focusing in nematic liquid crystals", Phys. Rev. A 48 (1993) 611-622.

6. M. Warenghem, J.F. Henninot, G. Abbate, "Bulk optical Frederiks effect: nonlinear optics of nematic liquid crystals in capillaries," Mol. Cryst. Liq. Cryst. 320 (1998) 207-230.

7. M. Warenghem, J.F. Henniot, G. Abbate, "Non linearly induced self waveguiding structure in dye doped nematic liquid crystals confined in capillaries," Opt. Express 2 (1998) 483-490.

8. M. Peccianti, A. De Rossi, G. Assanto, A. De Luca, C. Umeton, I.C. Khoo, "Electrically assisted selfconfinement and waveguiding in planar nematic liquid crystal cells", Appl. Phys. Lett. 77 (2000) 7-9.

9. M.A. Karpierz, M. Sierakowski, M. Świłło, T.R. Woliński, "Self focusing in liquid crystalline waveguides," Mol. Cryst. Liq. Cryst. 320 (1998) 157-164.

10. H. Lin, P. Palffy-Muhoray, M.A. Lee, "Liquid crystalline cores for optical fibers," Mol. Cryst. Liq. Cryst. 204 (1991) 189-200.

11. T.R. Woliński, A. Szymańska, T. Nasiłowski, M.A. Karpierz, A. Kujawski, R. Dąbrowski, "Propagation effects in liquid crystal-core optical fiber waveguides," Mol. Cryst. Liq. Cryst. 321 (1998) 113-124.

12. G. Abbate, F. Castaldo, L. De Stefano, "Nonlinear effects in liquid crystal waveguides: theory and experiment," Mol. Cryst. Liq. Cryst. 282 (1996) 269-286.

13. M.A. Karpierz, A.W. Domański, M. Sierakowski, M. Świłło, T.R Woliński, "Optical nonlinearity in liquid crystalline optical waveguides", Acta Phys. Polonica 95 (1999) 783-792.

14. C. Garcia-Reimbert, C. Garza-Hume, A.A. Minzoni, J.A. Reyes, R.F. Rodriguez, N.S. Smyth, "Active propagation and cut-off for low TM modes in a nonlinear nematic waveguide", Physica D 145 (2000) 144-157.

15. I. Janossy, "Molecular interpretation of the absorption induced optical reorientation of nematic liquid crystals," Phys. Rev. E 49 (1994) 2957-2963.

16. S. Gatz, J. Hermann, "Soliton propagation in materials with saturable nonlinearity", J. Opt. Soc. Am. B 8 (1991) 2296-2302.

17. D.W. McLaughlin, D.J. Muraki, M.J. Shelley, X. Wang, "A paraxial model for optical self-focussing in a nematic liquid crystal", Physica D 88 (1995) 55-81.

18. D.W. McLaughlin, D.J. Muraki, M.J. Shelley, "Self-focussed optical structures in a nematic liquid crystal", Physica D 97 (1996) 471-497.

19. F. Derrien, J.F. Henninot, M. Warenghem, G. Abbate, "A thermal (2D+1) spatial optical soliton in a dye doped liquid crystal", J. Opt. A - Pure Appl. Optics 2 (2000) 332-337. 